\title{
Cerebral Microbleeds Identified by Susceptibility-Weighted Imaging in Two Cases of Fabry Disease without Neurological Symptoms
}

\author{
Mineka Yamazoe1, Masayuki Maeda ${ }^{2 *}$, Maki Umino1, Hidekazu Tomimoto ${ }^{3}$, \\ Hajime Sakuma1 \\ ${ }^{1}$ Department of Radiology, School of Medicine, Mie University, Mie, Japan \\ ${ }^{2}$ Department of Advanced Diagnostic Imaging, School of Medicine, Mie University, Mie, Japan \\ ${ }^{3}$ Department of Neurology, School of Medicine, Mie University, Mie, Japan \\ Email: *mmaeda@clin.medic.mie-u.ac.jp
}

Received 12 October 2015; accepted 1 December 2015; published 4 December 2015

Copyright (C) 2015 by authors and Scientific Research Publishing Inc.

This work is licensed under the Creative Commons Attribution International License (CC BY). http://creativecommons.org/licenses/by/4.0/

(c) (7) Open Access

\begin{abstract}
Cerebrovascular disease is one of the fatal causes of Fabry disease (FD). Brain magnetic resonance imaging findings typically show lacunar infarcts in young patients with FD, but brain hemorrhages in FD are rarely reported. We report two cases of FD focusing on cerebral microbleeds (CMBs). Susceptibility-weighted imaging (SWI) and $\mathrm{T} 2 *$-weighted imaging reveal several lobar and deep CMBs in two patients with no medical history of stroke symptoms, hypertension, and anticoagulant/antiplatelet treatment. SWI can detect a greater number of CMBs than $\mathrm{T} 2 *$-weighted imaging. Thus, SWI is an excellent tool for identifying underlying CMBs in FD.
\end{abstract}

\section{Keywords}

Fabry Disease, Magnetic Resonance Imaging, Susceptibility-Weighted Imaging, Cerebral Microbleeds

\section{Introduction}

Fabry disease (FD) is a rare, $\mathrm{X}$-linked, inherited disorder of glycosphingolipid metabolism, which is caused by absent or deficient activity of $\alpha$-galactosidase A. There is resultant progressive accumulation of globotriaosylce-

\footnotetext{
${ }^{*}$ Corresponding author.
}

How to cite this paper: Yamazoe, M., Maeda, M., Umino, M., Tomimoto, H. and Sakuma, H. (2015) Cerebral Microbleeds Identified by Susceptibility-Weighted Imaging in Two Cases of Fabry Disease without Neurological Symptoms. Open Journal of Medical Imaging, 5, 194-198. http://dx.doi.org/10.4236/ojmi.2015.54024 
ramide (Gb3) within lysosomes in a variety of cell types, including endothelial, renal, cardiac, and dorsal root ganglion neuronal cells, with characteristic features such as acroparesthesia, hypohydrosis, angiokeratoma, and albuminuria. At a later age, progressive renal failure, hypertrophic cardiomyopathy, and cerebrovascular disease can occur [1].

Although cerebral infarction is recognized as an important manifestation of FD [2], cerebral hemorrhage seems to be underrecognized in this disorder. Moreover, regarding cerebral microbleeds (CMBs) identified by magnetic resonance imaging (MRI), only one study has reported using T2*-weighted images [3]. Susceptibility-weighted imaging (SWI) utilizes the susceptibility differences between adjacent tissues to produce tissue contrast and is exquisitely sensitive to the detection of blood products. To the best of our knowledge, there have been no reports on FD cases evaluated using SWI. We report two FD cases without neurological symptoms, focusing on CMBs identified using SWI.

\section{Case Report}

\subsection{Case 1}

The patient was a 42-year-old man with acroparesthesias, hypohydrosis, and angiokeratomas on the trunk, arms, and legs since childhood. The diagnosis of FD was confirmed based on clinical findings and decreased $\alpha$-galactosidase A activity at 24 years of age. Thereafter, he was followed up at a local hospital, and he underwent transthoracic echocardiography, 12-lead electrocardiography, and 24-h Holter monitoring once a year. He received neither enzyme replacement therapy nor anticoagulant/antiplatelet medications, but carbamazepine was prescribed for pain relief. Transthoracic echocardiography revealed diffuse, mild left ventricular hypertrophy at 42 years of age. The patient was admitted to our hospital for additional examinations, and his physical and neurological examinations, including blood pressure, were unremarkable. Blood tests were also unremarkable, whereas urinary sediment showed mulberry bodies. Electron microscopic examination of endomyocardial biopsies revealed lysosomal storage in the cardiomyocytes.

Brain MRI (3T, Ingenia; Koninklijke Philips N.V., Amsterdam, Netherlands) was performed, and no definite infarcts or high-signal lesions were found on fluid attenuation inversion recovery (FLAIR) or T2-weighted imaging. No high-signal abnormalities were apparent in the pulvinar on the T1-weighted images. No dolichoectasia of the vertebrobasilar arteries was visible on magnetic resonance angiography. However, SWI and T2*weighted imaging revealed several lobar and deep CMBs (Figure 1(a) \& Figure 1(b)), and SWI showed a greater number of CMBs. Enzyme replacement therapy was started after the MR examination.

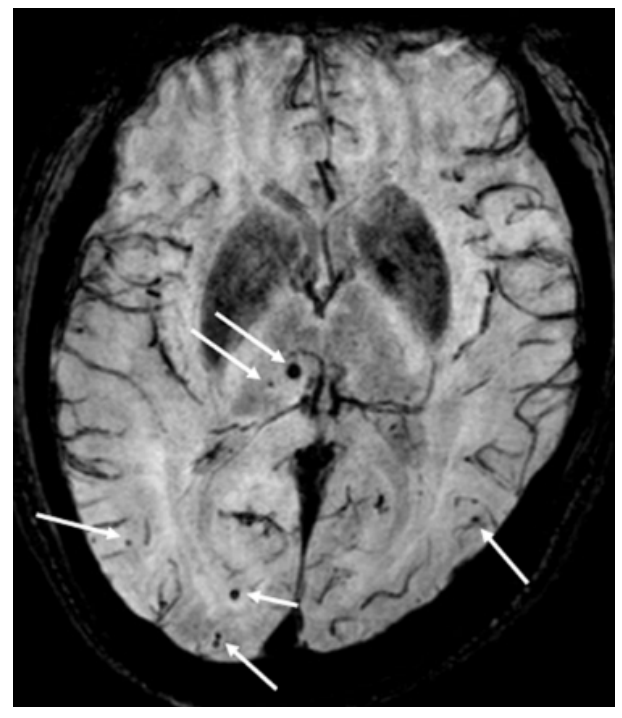

(a)

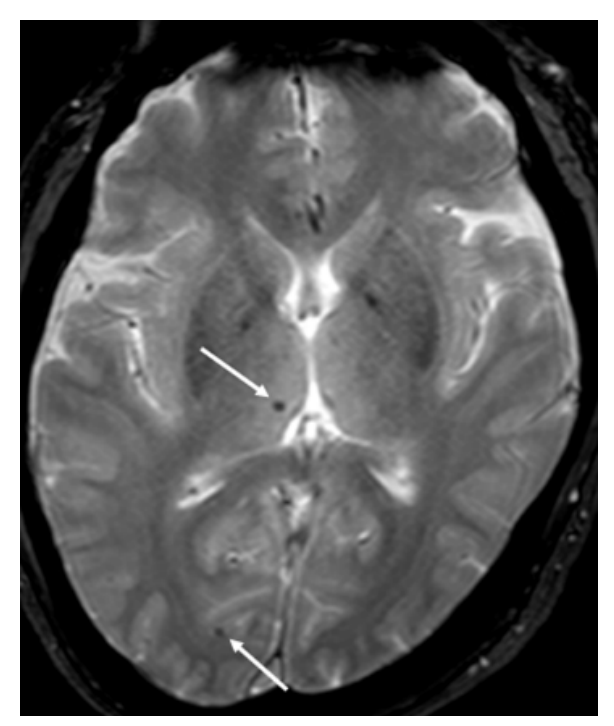

(b)

Figure 1. MRI in Case 1. SWI (a) and T2*-weighted images (b). SWI and T2*-weighted imaging revealed several CMBs in the cortex and the thalamus (arrows) ((a), (b)). SWI shows a greater number of CMBs compared with $\mathrm{T} 2 *$-weighted imaging (a). 


\subsection{Case 2}

The patient was a 45-year-old man and the brother of Case 1. He had the same symptoms (acroparesthesias, hypohydrosis, and angiokeratomas) as his younger brother and was suspected of having FD. The patient was admitted to our hospital for additional examinations. He had no medical history of enzyme replacement therapy or anticoagulant/antiplatelet treatment. No hypertension was observed, and the blood tests were also unremarkable. Like his brother, urinary sediment analysis showed mulberry bodies, and a hearing test revealed sensorineural hearing loss and vestibular function impairment. A cardiac MRI was performed, which revealed left ventricular wall thickening, and inferolateral myocardial fibrosis. Electron microscopic examination of the endomyocardial biopsies revealed lysosomal storage in the cardiomyocytes. The diagnosis of FD was confirmed based on clinical findings, pathologic findings, and decreased $\alpha$-galactosidase A activity.

Brain MRI (3T, Ingenia; Koninklijke Philips N.V.) was performed, which showed several old lacunar infarcts in the brain stem and cerebral white matter (Figure 2(a)). However, the patient had no history of stroke, indicating asymptomatic infarcts. FLAIR showed mild white matter lesions, and SWI revealed multiple lobar CMBs that were not seen using T2*-weighted imaging (Figure 2(b) \& Figure 2(c)). Enzyme replacement therapy was started after the MR examination.

\subsection{MRI Analysis of CMBs}

The number of CMBs was counted on both SWI and T2*-weighted images in the two patients. The CMBs were classified into lobar and deep/infratentorial types, and the results are shown in Table 1. SWI showed a greater number of CMBs than T2*-weighted imaging in the two patients, particularly in Case 2.

\section{Discussion}

Cerebrovascular disease is one of the potential complications of FD. Characteristic brain MRI findings in FD include multiple infarcts in young patients (mean age: 40 years), particularly lacunar infarcts [2]; white matter lesions, which can occur from an early age [4]; dolichoectasia of vertebrobasilar arteries [5]; and T1 high signals in the pulvinar [6]. Brain hemorrhage is rarely reported in FD. However, some investigators have reported that

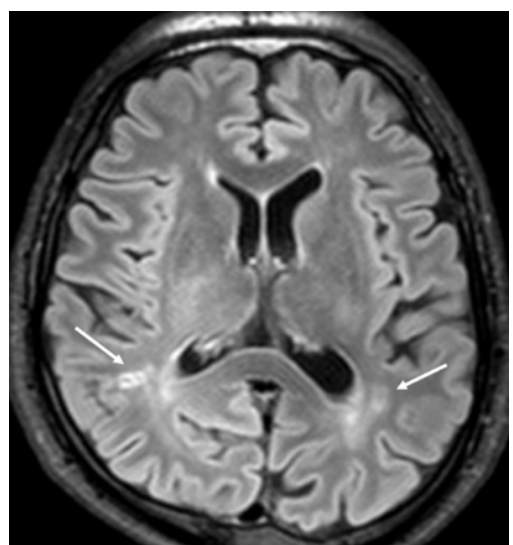

(a)

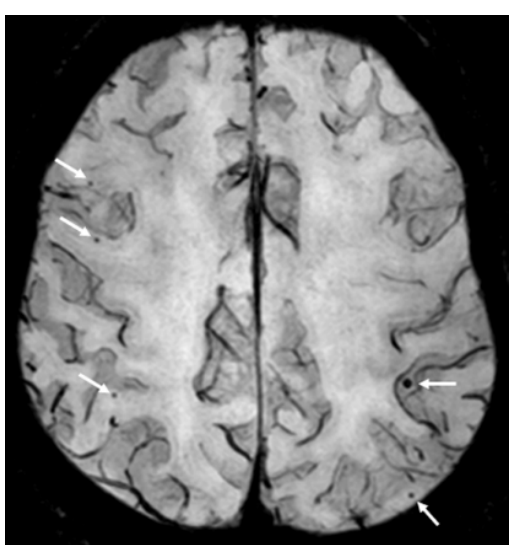

(b)

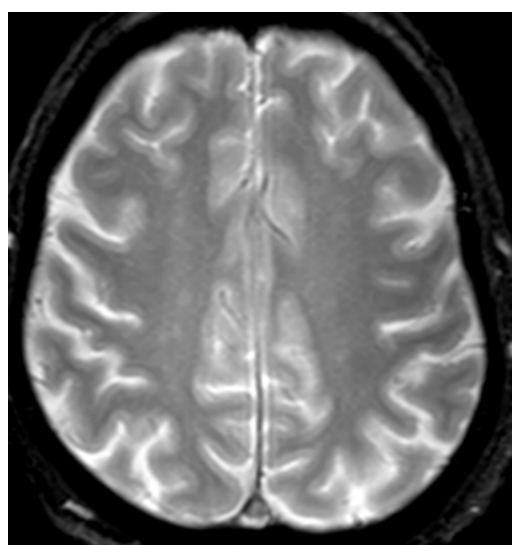

(c)

Figure 2. MRI in Case 2. FLAIR (a), SWI (b), and T2*-weighted images (c). FLAIR showed an old lacunar infarct in the cerebral white matter (arrows: (a)), whereas SWI revealed multiple lobar CMBs (arrows: (b)). However, none of these lesions were detected by $\mathrm{T} 2 *$-weighted imaging (c).

Table 1. Number of cerebral microbleeds detected on SWI and T2*-weighted imaging.

\begin{tabular}{|c|c|c|c|c|}
\hline & \multicolumn{2}{|l|}{ SWI } & \multicolumn{2}{|c|}{ T2*-Weighted Images } \\
\hline & Deep/Infratentorial & Lobar & Deep/Infratentorial & Lobar \\
\hline Case 1 & 9 & 20 & 2 & 6 \\
\hline Case 2 & 6 & 14 & 1 & 0 \\
\hline
\end{tabular}


T2*-weighted images show CMBs in $11 \%$ of neurologically asymptomatic FD patients [3]. On the other hand, no reports have described CMBs identified by SWI. Our results show that SWI detected a much greater number of CMBs in two patients with neurologically asymptomatic FD (Table 1). These results are not surprising because recent data have shown that SWI can detect significantly more CMBs (at least 67\% more) compared with conventional T2*-weighted imaging [7]. Additionally, due to its high sensitivity to hemosiderin, SWI can detect CMBs that are much smaller in size [8].

Reisin et al. reported that in three FD patients, all CMBs detected by $\mathrm{T}^{*}$-weighted imaging were located in deep cerebral areas such as the globus pallidus and thalamus [3]. Their three cases (aged 59 - 73 years) were older than our two cases, and two of them had hypertension and significant T2 hyperintense lesions [3]. The authors suggested that the CMBs in their cases resulted from hypertensive or atherosclerotic microangiopathy. It should be noted that in our two cases, there were two times more CMBs located in lobar areas than in deep/ infratentorial areas, which were detected by SWI (Table 1). This suggests that CMBs in FD may not always result from hypertensive or atherosclerotic microangiopathy. Larger prospective studies of MRI in FD using SWI are needed to confirm our findings.

A previous study indicated that most FD patients with hemorrhagic stroke are hypertensive (68.9\%) and that many patients present with both kidney and heart involvement [9]. Treatment with aspirin for secondary stroke prevention is reportedly another risk factor for hemorrhagic stroke in FD [10]. It remains uncertain if these risk factors are also applicable to CMBs in patients with FD. Our two patients had no history of hypertension or renal dysfunction and were not being treated with antiplatelet/anticoagulant medications. We speculate that the CMBs in our two patients were caused by small vessel impairment of the brain by Gb3 deposition. FD may have insidious CMBs even when patients present with no neurological symptoms or have no ischemic brain lesions.

\section{Conclusion}

SWI is extremely useful for detecting CMBs and may provide an insight into the pathophysiology of cerebrovascular complications in patients with FD.

\section{Conflict of Interest}

The authors have no conflicts of interest to declare.

\section{References}

[1] El-Abassi, R., Singhal, D. and England, J.D. (2014) Fabry’s Disease. Journal of the Neurological Sciences, 344, 5-19. http://dx.doi.org/10.1016/j.jns.2014.06.029

[2] Rolfs, A., Böttcher, T., Zschiesche, M., Morris, P., Winchester, B., Bauer, P., et al. (2005) Prevalence of Fabry Disease in Patients with Cryptogenic Stroke: A Prospective Study. The Lancet, 366, 1794-1796. http://dx.doi.org/10.1016/S0140-6736(05)67635-0

[3] Reisin, R.C., Romero, C., Marchesoni, C., Nápoli, G., Kisinovsky, I., Cáceres, G., et al. (2011) Brain MRI Findings in Patients with Fabry Disease. Journal of the Neurological Sciences, 305, 41-44. http://dx.doi.org/10.1016/j.jns.2011.03.020

[4] Fellgiebel, A., Müller, M.J. and Ginsberg, L. (2006) CNS Manifestations of Fabry’s Disease. The Lancet Neurology, 5, 791-795. http://dx.doi.org/10.1016/S1474-4422(06)70548-8

[5] Fellgiebel, A., Keller, I., Martus, P., Ropele, S., Yakushev, I., Böttcher, T., et al. (2011) Basilar Artery Diameter Is a Potential Screening Tool for Fabry Disease in Young Stroke Patients. Cerebrovascular Diseases, 31, $294-299$. http://dx.doi.org/10.1159/000322558

[6] Takanashi, J., Barkovich, A.J., Dillon, W.P., Sherr, E.H., Hart, K.A. and Packman, S. (2003) T1 Hyperintensity in the Pulvinar: Key Imaging Feature for Diagnosis of Fabry Disease. American Journal of Neuroradiology, 24, 916-921.

[7] Nandigam, R.N., Viswanathan, A., Delgado, P., Skehan, M.E., Smith, E.E., Rosand, J., et al. (2009) MR Imaging Detection of Cerebral Microbleeds: Effect of Susceptibility-Weighted Imaging, Section Thickness, and Field Strength. American Journal of Neuroradiology, 303, 38-43.

[8] Ayaz, M., Boikov, A.S., Haacke, E.M., Kido, D.K. and Kirsch, W.M. (2010) Imaging Cerebral Microbleeds Using Susceptibility-Weighted Imaging: One Step toward Detecting Vascular Dementia. Journal of Magnetic Resonance Imaging, 31, 142-148. http://dx.doi.org/10.1002/jmri.22001

[9] Sims, K., Politei, J., Banikazemi, M. and Lee, P. (2009) Stroke in Fabry Disease Frequently Occurs before Diagnosis 
and in the Absence of Other Clinical Events: Natural History Data from the Fabry Registry. Stroke, 40, 788-794. http://dx.doi.org/10.1161/STROKEAHA.108.526293

[10] Nakamura, K., Sekijima, Y., Nakamura, K., Hattori, K., Nagamatsu, K., Shimizu, Y., et al. (2010) Cerebral Hemorrhage in Fabry’s Disease. Journal of Human Genetics, 55, 259-261. http://dx.doi.org/10.1038/jhg.2010.18 